\title{
Brain infarction after cervical spine fracture due to vertebral artery injury
}

\begin{abstract}
Ischemic strokes may occur after cervical spine injuries, such as fracture, dislocation, or spinal cord injury. In fact, cervical spine injury is a risk factor for blunt vertebral artery injury. Although several lines of evidence indicate an increased risk of stroke after cervical spine injury, its pathologic mechanism remains largely unknown. The neurologic symptoms due to VAI occlusion usually manifest within the first day after cervical trauma. Bilateral VA injuries can lead to severe vertebrobasilar insufficiencies and occur with shorter latencies than those of unilateral occlusions. Unilateral VA occlusions rarely result in neurological deficits because of the collateral blood supplies delivered by the contra lateral VA and the posterior inferior cerebella artery. We report a rare case of a 64-year-old man presented in the emergency department with a $\mathrm{C} 3 / \mathrm{C} 4$ subluxation and unilateral complete vertebral artery occlusion that resulted from a motor vehicle accident. After surgery, his deficits where initially improved, but 15 hours later he suddenly presented with headache, clumsiness in handling objects, right hemianospia and his motor weakness was aggravated. Cranial CT revealed the presence of a stroke in the left occipital lobe. We immediately treated the patient with an anticoagulation therapy. Nine months later his weakness had partially improved.
\end{abstract}

Keywords: cervical spine trauma, vertebral artery injury, cerebral infarction, fracture, management

\author{
Volume 8 Issue 6 - 2018
}

\author{
Bougiouklis Dimitrios,' Gantaifis Nikolaos,' \\ Rovlias Aristides ${ }^{2}$ \\ 'Orthopaedic Department of General Hospital of Pyrgos, \\ Syntriada Pyrgos, Greece \\ ${ }^{2}$ Neurosurgery Department of General Hospital “Asklepieion” \\ Voulas, Athens, Greece
}

Correspondence: Bougiouklis Dimitrios MD, Orthopaedic Department of General Hospital of Pyrgos, Syntriada Pyrgos, Greece, 27I00, Tel 003026210 82706, 00302623 40I67I, 0030 699 3390492, Email dinmed@hotmail.com

Received: February 0I, 2018 | Published: November 29, 2018
Abbreviations: VAI, vertebral artery injury; ATLS, advanced trauma life support; CT, computed tomography; DSA, digital subtraction angiography; MRA, magnetic resonance angiography; CTA, computed tomography angiography

\section{Introduction}

Vertebral artery injuries after blunt cervical trauma are common. Hyperextension injuries, with or without lateral flexion and rotation, have previously been discussed as the most common mechanism of closed injury to the VA. ${ }^{1}$ VAI can be unilateral or bilateral. Bilateral VAI is less frequent. Unilateral occlusion of the vertebral artery seldom results in a neurological deficit if the collateral supply through the other vertebral and posterior inferior arteries is sufficient. Bilateral occlusion may also be asymptomatic. However, this usually causes neurological deficit or even death. ${ }^{2}$ VAI has been under diagnosed or misdiagnosed frequently. The vertebral is most susceptible to injury at the point of entrance into the transverse foramen of $\mathrm{C} 6$. The second most common site of VAI is at $\mathrm{C} 1-\mathrm{C} 2 .{ }^{3}$ Ischemic stroke after cervical spine trauma depends on the severity of trauma, the patient's age and his comorbidities. ${ }^{4}$ The rationale for screening of asymptomatic trauma patients for the presence of VAI is that it may occur in many asymptomatic patients and anticoagulation may prevent strokes and improve neurological outcome. We presented a rare case of a 64-yearold man which he sustained a $\mathrm{C} 3 / \mathrm{C} 4$ subluxation that demanded operative reduction. 15hours after surgery he presented with left occipital lobe infarction due to unilateral vertebral artery occlusion.

\section{Case presentation}

A 64-year-old man, with diabetes mellitus type II and dyslipidemia, presented to the emergency department because of a motor vehicle accident. He was the driver, not having on the seatbelt. During examination, based on protocol ATLS, the patient alert and fully oriented, his vital signs were stable, but he complained of neck pain and quadriparesis. In the right upper extremity was noted a weakness equal to $3 / 5$, while in the left upper extremity was noted a weakness equal to $1 / 5$. The weakness of his lower extremities was rated as grade 4/5. An initial cranial CT was normal. X-ray and CT scan of the cervical spine revealed a subluxation at the $\mathrm{C} 3 / \mathrm{C} 4$ level (Figure 1). Furthermore, MRI of cervical spine took place which demonstrated a complete occlusion of left vertebral artery at the $\mathrm{C} 3 / \mathrm{C} 4$ level (Figure 2).

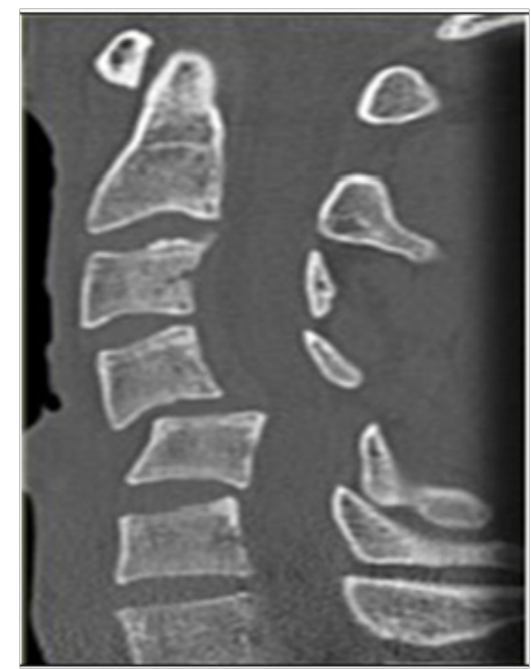

Figure I CT scan of cervical spine show the C3/C4 subluxation. 
Due that, the patient was transferred to operation theatre, where we performed $\mathrm{C} 3 / \mathrm{C} 4$ discectomy and stabilization with a plate using the anterior approach, and a C3-C4 decompressive laminectomy and C3-C4 fixation with screws using the posterior approach. During the first post-operative day the patient was hemodynamically stable and his upper extremity weakness improved from grade $3 / 5$ to $5 / 5$ in the right, and from grade $1 / 5$ to $3 / 5$ in the left. However, 15 hours later, he suddenly presented with headache, clumsiness in handling objects, right hemianospia. His right upper extremity strength also deteriorated from grade $5 / 5$ to $2 / 5$. Because of that, cranial CT was carried out which revealed an infraction in the left occipital lobe (Figure 3). Immediately, we initiated anticoagulation therapy and in the next days patient's motor weakness gradually improved. Nine months later the motor strength of both upper and lower extremities had partially improved and the patient was able to walk with a cane.

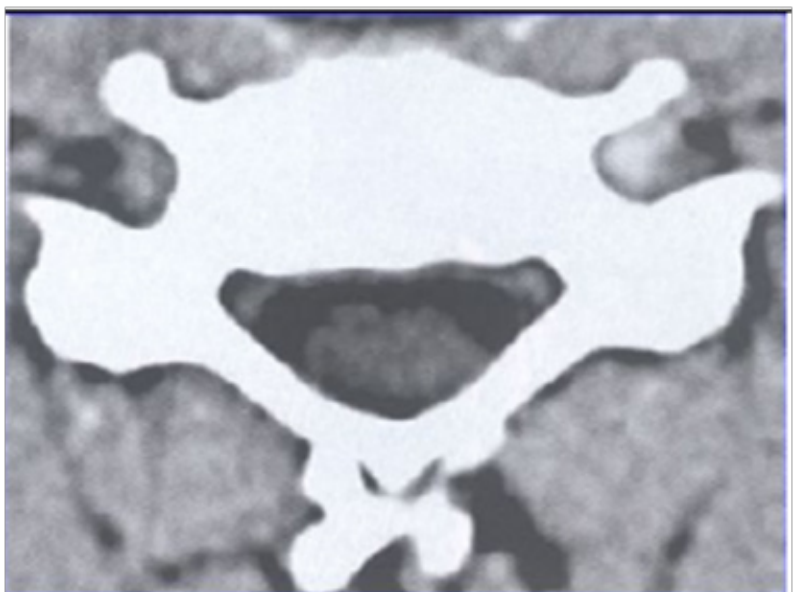

Figure 2 Pre-operative MRI revealed a complete occlusion of left vertebral artery at the $\mathrm{C} 4$ livel in the axial view.

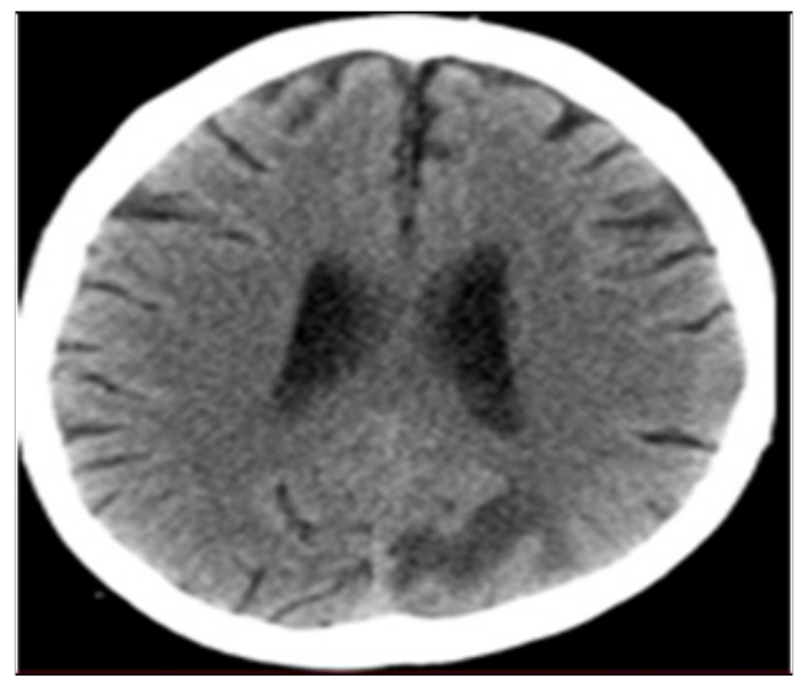

Figure 3 The first post-operative day a cranial CT demonstrated an ifranction in the left occipital lobe.

\section{Discussion}

Ischemic stroke is not uncommon after cervical spine injury. Indeed, according $\mathrm{H}$ Chikuda et $\mathrm{al},{ }^{5}$ an ischemic stroke after cervical spine injury occurs in $1 \%$ of patients during hospitalization with a threefold increase in mortality. ${ }^{5}$ Regarding pathomechanism, previous studies have demonstrated that cervical spine injury is a risk factor for blunt cerebrovascular injury, especially VAI. In the literature, the incidence of VAI in cervical spine trauma ranges from 3 to $39 \%$. However VAI has been underdiagnosed or misdiagnosed frequently. This mainly occurs because most VAI patients remain asymptomatic if the vertebral artery is damaged only unilaterally. DeSouza MR et al refered that only $20 \%$ of patients with a unilateral VAI after cervical trauma, develops neurologic symptoms. ${ }^{7}$ Furthermore, vertebral artery occlusion is rarely symptomatic because of collateral blood supply from the contralateral vertebral artery and the circle of Willis. ${ }^{8}$ Nevertheless, misdiagnosed VAI has often been reported to cause acute neurologic deterioration of previously conscious patients with cervical spine injury. Unlike unilateral VA occlusion in which only $20 \%$ of patients are symptomatic, most patients with bilateral VA occlusion are symptomatic. Patients with bilateral VAI usually presented with more severe symptoms, including an altered mental status, pinpoint pupils, and even sudden respiratory arrest. ${ }^{9}$

Three types of cervical fractures are associated with significant risk for VAI: fractures involving the transverse foramen, subluxations and fractures involving the upper cervical spine (C1-C3). In a study of Vaccaro et al. ${ }^{10}$ the incidence of VAI in fracture dislocations and subluxations of cervical spine was $40 \% .^{10}$ Cothen reported an $18 \%$ incidence of VAI in fractures of the upper cervical spine (C1-C3). ${ }^{11}$ The VA is most susceptible at the C5-C6 level just after it enters the transverse foramen. Other areas of susceptibility are located at the atlanto-axial level just after the VA leaves the transverse foramen, and at the atlanto-occipital level where the VA is tethered by the dura. Ozveren et al. ${ }^{12}$ mentioned that the mobility of the artery suddenly decreases as it enters the transverse foramen of C-6. Hyperextension injuries, with or without lateral flexion and rotation, have previously been accepted as the most common mechanism of closed injury to the vertebral artery. ${ }^{12}$ Suggested VAI should be suspected in any patient who presents with hemorrhage from the mouth, nose, or ear and any mechanism of injury significant for severe cervical hyperextension/ rotation or hyper flexion. The mechanism responsible for VA injury has been suggested to be stretching, tearing of the intima and media, or compression of the vessels. Motor vehicle accidents are the leading cause of VA injury due to the major rotational component. Rotational forces applied to the mobile portions of the cervicocephalic arteries are responsible for intimal tears.

The incidence of ischemic stroke following VAI depends on both the grade of VAI (intimal flaps, dissection, pseudoaneurism, complete occlusion) and on the age of the patients and co morbidities. In addition, the size of the contra lateral VA, the completeness of the circle of Willis, and the robustness of the native pial collateral seem to be important factors affecting the development of infarction after VAI. Patients with VAI have a significantly higher rate of strokes than those without. The in-hospital stroke rate increased with advancing age, diabetes, heart disease, and worse consciousness level at admission. ${ }^{13}$

VAI do not always cause ischemic symptoms immediately after the trauma. Almost half cases it is noticed an interlude of at least $18 \mathrm{~h}$ between the trauma and the onset of ischemic symptoms. It has been suggested that intimal tears of vertebral artery allows blood under arterial pressure to enter between the layers of the wall of the artery, forming an intramural hematoma. Therefore this can lead to the aggregation of platelets on the exposed sub-intimal tissue and subsequent thrombosis, clot propagation and/or embolization to the 
cerebrum. The interludes are likely due to the slow progression of thrombosis or the gradual swelling of the vessel. In our case, the left occipital lobe infarction was likely the result of an embolus, rather than thrombus formation. Emboli in the basilar territory do not commonly cause extensive brain stem infarction because an embolus of sufficient size to traverse the VA would be unlikely to occlude the larger diameter basilar artery. ${ }^{14,15}$ We propose that the left occipital lobe lesion was likely due to an embolus from the VA occlusion site because there was no infarction in the brain stem.

Improper immobilization of cervical spine during the patient's transport may increase subluxation and this fact may further worsen VAI. For that reason it is important the proper immobilization and good neck positioning. Depending on the mechanism and morphology of cervical spine injury, proper immobilization could come in different forms. It could be argued that an injury of this severity it is extremely necessary a halo-vest placement especially when long-distance transfers are needed. ${ }^{16}$

X-rays and CT scans identify $98 \%$ of cervical fractures and $99 \%$ of subluxations and are justified diagnostic tool in cervical spine trauma. There have been no definite standards or guidelines as to what is the most important and optimal imaging study for patients suspected of VAI. Being that digital DSA is the criterion standard, neither MRA nor CTA has been able to show comparable sensitivity and specificity. Initial use of contrast enhanced CT scans and/or MRI scans may be wise during initial trauma patient evaluation with severe cervical hyperextension/rotation or hyperflexion. ${ }^{17,18}$

In unstable cervical spine fractures, to prevent further VAI damage, early surgical fixation is considered to be indicated. Optimal medical treatment of VAI is still under debate and an evidence base of anticoagulation in patients with VAI does not exist. However, until now anticoagulative treatment is a standard therapy. Previous studies showed a decreased stroke rate from $30-50$ to $2-10 \%$ using anticoagulation or antiplatelet therapy. ${ }^{19}$

\section{Conclusion}

Cervical spine trauma may cause vertebral artery injuries which lead in unilateral or bilateral occlusions. The first one rarely causes neurological symptoms due to the existence of the collateral channels. Therefore, in any case the type of cervical injury, the patient's age and his co morbidities should be estimated. Initial use of contrast enhanced CT scans and/or MRI scans may be wise during initial trauma patient evaluation with severe cervical hyperextension/rotation or hyper flexion. In unstable cervical fractures accompanied by VAI, after their surgical fixation, it is reasonable the beginning of an anticoagulation therapy.

\section{Acknowledgments}

None.

\section{Conflicts of interest}

Authors declare no conflict of interest or any funding source received for this study.

\section{References}

1. Friedman D, Flanders A, Thomas C, et al. Vertebral artery injury after acute cervical spine trauma: rate of occurrence as detected by MR angiography and assessment of clinical consequences. AJR Am J Roentgenol. 1995; 164:443-447.
2. Harrop JS, Sharan AD, Vaccaro AR, et al. The cause of neurologic deterioration after acute cervical spinal cord injury. Spine. 2001;26(4):340346.

3. Pelkonen O, Tikkakoski T, Leinonen S, et al. Extracranial internal carotid and vertebral artery dissections: angiographic spectrum, course and prognosis. Neuroradiology. 2003;45(2):71-77.

4. Stein DM, Boswell S, Sliker CW, et al. Blunt cerebrovascular injuries: does treatment always matter? J Trauma. 2009;66(1):132-143.

5. Chikuda H, Yasunaga H, Horiguchi H, et al. Mortality and morbidity in dialysis-dependent patients undergoing spinal surgery: analysis of a national administrative database in Japan. J Bone Joint Surg Am. 2012;94(5):433-438.

6. Biffl WL, Moore EE, Elliott JP, et al. The devastating potential of blunt vertebral arterial injuries. Ann Surg. 2000;231(5):672-681.

7. Desouza RM, Crocker MJ, Haliasos N, et al. Blunt trau $\neg$ matic vertebral artery injury: a clinical review. Eur Spine J. 2011;20(9):1405-1416.

8. Chung D, Sung JK, Cho DC, et al. Vertebral artery injury in desta $\neg$ bilized midcervical spine trauma; predisposing factors and proposed mechanism. Acta Neurochir (Wien). 2012;154(11):2091-2098.

9. Taylor MW, Senkowski CK. Bilateral vertebral artery dissection after blunt cervical trauma: case report and review of the literature. $J$ Trauma. 2002;52(6):1186-1188.

10. Vaccaro AR, Klein GR, Flanders AE, et al. Long-term evaluation of vertebral artery injuries following cervical spine trauma using magnetic resonance angiography. Spine. 1998;23(7):789-794.

11. Cothren CC, Moore EE, Biffl WL, et al. Cervical spine fracture patterns predictive of blunt vertebral artery injury. $J$ Trauma. 2003;55(5):811813.

12. Ozveren F, Ziyal IM, Bejjani GK, et al. Bilateral vertebral artery occlusion following cervical spine trauma - case report. Neurol Med Chir (Tokyo). 1999;39(1):28-32.

13. Lebl DR, Bono CM, Velmahos G, et al. Vertebral artery injury associated with blunt cervical spine trauma : a multi-variate regression analysis. Spine. 2013;38(16):1352-1361.

14. Mueller CA, Peters I, Podlogar M, et al. Vertebral artery injuries following cervical spine trauma : a prospective observational study. Eur Spine J. 2011;20(12):2202-2209.

15. Muratsu H, Doita M, Yanagi T, et al. Cerebellar infarction resulting from vertebral artery occlusion associ-ated with a Jefferson fracture. $J$ Spinal Disord Tech. 2005;18(3):293-296.

16. Hauswald M, Ong G, Tandberg D, et al. Out-of-hospital spinal immobilization: its effect on neurologic injury. Acad Emerg Med. 1998;5(3):214-219.

17. Miller PR, Fabian TC, Croce MA, et al. Prospective screening for blunt cerebrovascular injuries: analysis of diagnostic modalities and outcomes. Ann Surg. 2002;236:386-395.

18. Hollingworth W, Nathens AB, Kanne JP, et al. The diagnostic accuracy of computed tomography angiography for traumatic or atherosclerotic lesions of the carotid and vertebral arteries: a systematic review. Eur $J$ Radiol. 2003;48(1):88-102.

19. Cothren CC, Moore EE, Biffl WL, et al. Anticoagulation is the gold standard therapy for blunt carotid injuries to reduce stroke rate. Arch Surg. 2004;139(5):540-545. 